\title{
AFECTACIÓN GANGLIONAR EN PACIENTE CON ENDOMETRIOSIS PROFUNDA RECTOVAGINAL
}

\author{
Israel Ortega Sánchez ${ }^{1}$, Bárbara Castro Martín ${ }^{1}$, Javier de Santiago García ${ }^{1}$, Alicia \\ Hernández Gutiérrez ${ }^{1}$, Dulce Mํㅡㄹ Benito López ${ }^{2}$, Juan Ordás Santo Tomás ${ }^{1}$. \\ 1 Departamento de Obstetricia y Ginecología, Unidad de Endometriosis; ${ }^{2}$ Departamento de Anatomía Patológica, \\ Hospital Universitario La Paz, Madrid, España.
}

\section{RESUMEN}

Se describe un raro caso de endometriosis rectovaginal con compromiso ganglionar en mujer de 33 años. La presencia de tejido endometrial en ganglios linfáticos pélvicos es rara y ha sido confirmada en la literatura en mujeres que han sido sometidas a cirugía por endometriosis. La presencia de endometriosis en los ganglios linfáticos pélvicos es muy improbable que surja de novo y sugiere extensión de la enfermedad.

\section{PALABRAS CLAVE: Endometriosis profunda, infiltración ganglionar}

\section{SUMMARY}

A rare case of rectovaginal endometriosis with lymph node involvement is described in a 33-year-old patient. The presence of endometrial tissue in pelvic lymph nodes is rare and has been confirmed in the literature in subjects who underwent surgery for endometriosis. Involvement of pelvic lymph nodes by endometriosis seems unlikely to arise de novo and probably suggests lymphatic spread of the disease.

\section{KEY WORDS: Deep endometriosis, lymph node involvement}

\section{INTRODUCCIÓN}

La diseminación linfática de la endometriosis fue observada por primera vez en 1925 (1). La presencia de tejido endometrial en ganglios linfáticos pélvicos es excepcional y ha sido recogida en la literatura en pacientes que fueron sometidas a cirugía por endometriosis (2-4). La afectación de los ganglios pélvicos por endometriosis, parece improbable que aparezca de novo y sugiere un extensión linfática de la enfermedad (2-5).

\section{Caso clínico}

Paciente de 33 años que fue remitida a la Unidad de Endometriosis del Hospital Universitario de La Paz, España, por diagnóstico de endometriosis profunda. Refería dismenorrea intensa y progresiva desde hacía 15 años, que había requerido tratamiento con análogos GnRH y anticonceptivos hormonales orales, empeorándose su estado en el último año con estreñimiento, continuos cuadros pseudoobstructivos, disquecia, importante pérdida 
de peso y en los últimos cinco meses rectorragia coincidente con la menstruación.

Se remite a la paciente a nuestro centro 10 días después de haberle practicado una primera laparotomía, con diagnóstico de endometriosis profunda y con el fin de realizar una cirugía completa. Durante esa primera cirugía se realizó liberación de adherencias a nivel de anexo izquierdo, decapsulación del quiste anexial derecho, citología de lavado peritoneal y biopsias de serosa peritoneal. Se decidió tratamiento conservador de manera intraoperatoria, dada la proximidad de la estenosis rectal al margen anal y al carácter benigno en los hallazgos anatomopatológicos.

La exploración en consulta revelaba un nódulo empastado en el tabique rectovaginal, mientras que la ecografía abdominal mostraba útero y anexos normales. La analítica de sangre reveló ligera anemia.

Se decide ingreso y programación de cirugía una semana más tarde. En el transcurso de la misma se evidencia útero en retroflexión y saco de Douglas ocupado por ambos anexos y recto-sigmoides íntimamente adherido a cara posterior uterina. Quiste endometriósico en ovario izquierdo, siendo el derecho rigurosamente normal. Gran foco de endometriosis profunda que infiltra recto a $5 \mathrm{~cm}$ del margen anal, con afectación de parametrio derecho y fascia presacra.

Se efectúa laparotomía, realizando quistectomía izquierda, salpingectomía bilateral, apendicectomía, adhesiolisis y liberación de sigmoides y colon, disección e identificación de ambos uréteres hasta el túnel de los mismos y posterior disección de ambas fosas pararrectales. Se procede a la extirpación del nódulo endometriósico, así como de un implante en plica vesicouterina y de una adenopatía pélvica derecha aumentada de tamaño, con resección posterior de aproximadamente $20 \mathrm{~cm}$ de recto-sigmoides y anastomosis término-terminal con endorresector circunferencial.

El informe anatomopatológico reveló la existencia de focos de endometriosis a nivel de plica vesicouterina, serosa apendicular y de ambas trompas, así como de afectación de serosa y capas musculares de intestino grueso, sin alcanzar la mucosa. También se informó de la presencia de endometriosis quística en el ganglio linfático pélvico derecho extraído durante la cirugía.

Macroscópicamente se trataba de una formación nodular de $1,2 \mathrm{~cm}$ de diámetro máximo, con superficie externa pardusca y lisa. Al corte muestra un quiste de $8 \mathrm{~mm}$ con contenido gelatinoso. Microscópicamente se objetiva tejido linfoide rechazado a la periferia por un quiste (Figura $1 \mathrm{~A}$ ) revestido por epitelio cilíndrico de tipo endometrial, rodeado de estroma formado por células estrelladas (Figura $1 \mathrm{~B}$ ) y de glándulas de pequeño tamaño (Figura 1C).

La evolución postoperatoria fue satisfactoria con una marcada disminución de la dismenorrea, que cede con reposo y antiinflamatorios, al mismo tiempo que una progresiva desaparición de la disquecia y rectorragias, éstas últimas en relación con episodios de estreñimiento esporádicos y de corta duración.

\section{DISCUSIÓN}

La endometriosis es considerada como una enfermedad benigna, progresiva, que puede cursar con recurrencias y con invasión local y a distancia. Generalmente afecta a mujeres en edad reproductiva y es una de las causas más comunes de dolor pélvico y de infertilidad.

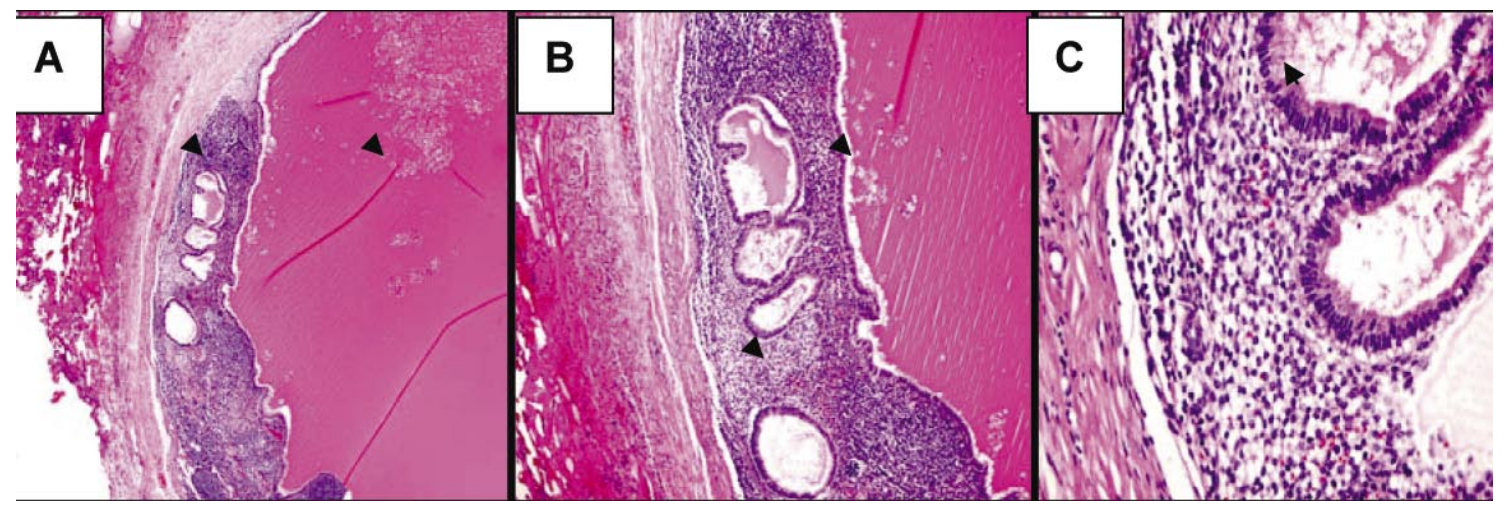

Figura 1. Tejido linfoide rechazado a la periferia por un quiste $(A)$ revestido por epitelio cilíndrico de tipo endometrial, rodeado de estroma formado por células estrelladas (B) y de glándulas de pequeño tamaño (C). 
Puede afectar localmente a estructuras pélvicas y áreas del peritoneo próximas a los ovarios, como los ligamentos uterosacros y fondo de saco de Douglas, o bien extenderse más allá del aparato genital, hecho que ocurre en aproximadamente un $12 \%$ de los casos. Las localizaciones extragenitales por orden de frecuencia son el intestino, el colon sigmoide, tracto urinario, apéndice, íleon y diafragma. Otras localizaciones que también pueden verse son la vulva, vagina, cérvix, tabique rectovaginal, pared abdominal, región umbilical, bronquios, pulmones, riñones, región inguinal, piel y sistema nervioso.

Aunque la etiología de la endometriosis no ha sido todavía claramente establecida, existen numerosas teorías que tratan de explicar la histogénesis de esta compleja enfermedad, como son la teoría de la menstruación retrógrada, la diseminación hematógena y linfática, la implantación directa y la metaplasia del epitelio celómico.

Lo particular de nuestro caso es la afectación de ganglios linfáticos en el desarrollo evolutivo de una endometriosis, hecho muy poco común y que en la mayoría de los casos recogidos en la bibliografía corresponden a ganglios afectados por estroma endometrial benigno que están desprovistos de anormalidades müllerianas, tales como los quistes müllerianos o endosalpingiosis. Esto último ocurre con una frecuencia del $2-41 \%$, y generalmente son hallazgos microscópicos que son encontrados en el marco de una salpingitis, tumores serosos borderline de ovario o carcinomas (6-9).

Algunos investigadores sostienen que existen principalmente dos hipótesis a la hora de explicar la afectación linfática por endometriosis. La primera considera que la afectación de los ganglios linfáticos se produce como consecuencia de un proceso de metaplasia del sistema mülleriano secundario (10-11). La segunda, en cambio, mantiene que este fenómeno es secundario al drenaje linfático del tejido endometrial (12-14).

En este sentido, se han definido cinco rutas diferentes de drenaje linfático con origen en el útero: (a) desde la matriz a los ganglios de la ilíaca interna y común, aórticos a través del ligamento infundibulopélvico, (b) desde las cadenas del obturador, ilíacos internos y externos hasta los ganglios de la cadena ilíaca común a través del ligamento cardinal, (c) a las cadenas ganlionares inguinales por medio de los ligamentos redondo y umbilical, (d) directamente hacia el ovario, (e) directamente hasta las trompas. Es interesante ver cómo estas rutas coinciden con los lugares de difusión de metástasis del cáncer de endometrio.

Algunos autores han intentado relacionar las características clínicas y anatomopatológicas de la endometriosis profunda y el grado de afectación linfática de la misma. Abrao y cols (15), estudiaron a 35 pacientes con endometriosis intestinal y observaron que en todos aquellos casos en los que el espesor de la lesión alcanzaba los $1,75 \mathrm{~cm}$ o afectaba a más del $80 \%$ de la circunferencia de la luz, los ganglios linfáticos aparecían infiltrados. No vieron relación alguna con la edad, síntomas clínicos o longitud de la lesión en la pared intestinal

Además se ha valorado la posible invasión linfovascular de glándulas y/o estroma en endometriosis de recto-sigmoides mediante el uso de inmunohistoquímica y el anticuerpo D2-40, con el fin de verificar la hipótesis de que la afectación linfática puede derivarse del drenaje vascular. Se ha demostrado que D2-40 reacciona con los epítopos glicosilados y no glicosilados de gp36 y es idéntico a la podoplanina, siendo un marcador muy sensible para el endotelio linfovascular, lo que ha sido ampliamente utilizado para identificar invasión linfovascular en carcinomas de mama, colon, próstata, cérvix y endometrio (16-18). En nuestro caso esta tinción no aportó información adicional.

A pesar de que la endometriosis es un proceso benigno, es cierto que en determinadas ocasiones puede compartir características de malignidad, como son la morfología anormal, un patrón de crecimiento celular alterado, invasión celular local y a distancia y neoangiogénesis. Existen estudios que demuestran cómo en el epitelio glandular ocasionalmente pueden desarrollarse aneuploidías en el DNA. Por otro lado, la experimentación in vitro sugiere que la endometriosis puede tener un origen monoclonal (19-21).

En nuestro caso, y teniendo en cuenta el avanzado grado de extensión local y linfática de la enfermedad, sería razonable plantearse si se considera todavía una enfermedad clínicamente benigna o podríamos hablar, por el contrario, de una endometriosis intestinal con hallazgos que sugieren premalignización.

En esta línea se ha demostrado que el componente predominante en las lesiones endometriósicas intestinales es el estroma, y que en caso de transformación maligna, éste se convertiría en un tumor de tipo sarcomatoso, cuyo pronóstico casi nunca es fatal. De hecho estas lesiones de endometriosis infiltrativa muestran similitudes con el sarcoma estromal de bajo grado en cuanto a la morfología celular, patrón de infiltración, expresión de receptores hormonales, lo que aumenta la posibilidad de que exista una relación entre las dos enfermedades similar a la observada entre fibromatosis (enfermedad localmente agresiva que no tiene potencial metastásico) y el fibrosarcoma (22-25). 
Finalmente pensamos que la realización de más estudios clínicos y anatomopatológicos proporcionarían la información necesaria como para poder establecer una relación entre endometriosis pélvica, diseminación de la misma a través de las distintas vías linfáticas y afectación de los ganglios linfáticos pélvicos.

\section{BIBLIOGRAFÍA}

1. Halban J. Metastatic hysteradenosis: lymphatic origin of so-called heterotropic adenofibromatosis. Arch Gynäk 1925;125:475-9.

2. Javert CT. Pathogenesis of endometriosis based on endometrial homeoplasia, direct extension, exfoliation and implantation, lymphatic and hematogenous metastases (including five case reports of endometrial tissue in lymph nodes). Cancer 1949;2:399-410.

3. Taussig FJ. Ectopic deciduas formation. Surg Gynecol Obstet 1906;2:292-3.

4. Russel HB. Decidual reaction of endometrium ectopic in an abnormal lymph node. Surg Gynecol Obstet 1945;81:218-20.

5. Moore JG, Binstock MA, Growdon WA. The clinical implications of retroperitoneal endometriosis. Am J Obstet Gynecol 1988;158:1291-8.

6. Horn LC, Bilek K. Frequency and histogenesis of pelvic retroperitoneal lymph node inclusions of the female genital tract. An immunohistochemical study of $34 \mathrm{ca}-$ ses. Pathol Res Pract 1995;191:991-6.

7. Maassen V, Hiller K. Glandular inclusions in lymph nodes pattern of distribution and metaplastic transformation. Arch Gynecol Obstet 1994;255:1-8.

8. Prade M, Spatz A, Bentley R. Borderline and malignant serous tumor arising in pelvic lymph nodes: evidence of origin in benign glandular inclusions. Int $\mathrm{J}$ Gynecol Pathol 1995;14:87-91.

9. Kheir SM, Mann WJ, Wilkerson JA. Glandular inclusions in lymph nodes.

The problem of extensive involvement and relationship to salpingitis. Am J Surg Pathol 1981;5:353-9.

10. Lauchlan SC. The secondary mullerian system. Obstet Gynecol Surv 1972;27:133-46.

11. Fergusson BR, Bennington JL, Haber SL. Histochemistry of mucosubstances and histology of mixed mullerian pelvic lymph node glandular inclusions. Evidence for histogenesis by mullerian metaplasia of coelomic epithelium. Obstet Gynecol 1969;33:617-25.

12. Halban Y. Hysteroadenosis metastica. Archive fur Gynakologie 1925;124:457-82.

13. Javert CT. Pathogenesis of endometriosis based upon endometrial homeoplasia, direct extension, exfoliation and implantation, lymphatic and hematogenous metastasis (including five case reports of endometrial tissue in pelvic lymph nodes). Cancer 1949;2:399-410.

14. Ueki M. Histologic study of endometriosis and examination of lymphatic drainage in and from the uterus. Am J Obstet Gynecol 1991;165:201-9.

15. Abrao MS, Podgaec S, Dias JA Jr, Averbach M, Garry R, Ferraz Silva LF, Carvalho FM. Deeply infiltrating endometriosis affecting the rectum and lymph nodes. Fertil Steril 2006;86(3):543-7.

16. Arnaout-Alkarain A, Kahn HJ, Narod SA, Sun $P$, Marks AN. Significance of lymph vessel invasion identified by endothelial lymphatic marker D2-40 in node negative breast cancer. Mod Pathol 2007;20:183-91.

17. Fernandez-Aguilar S, Jondet M, Simonart T, Noel JC. Microvessel and lymphatic density in tubular carcinoma of the breast: a comparative study with invasive low-grade ductal carcinoma. Breast 2006;15:782-5.

18. Watari H, Todo Y, Todeka M, Ebina Y, Yamamoto R, Sakuragi N. Lymph-vascular space invasion and number of positive para-aortic node groups predict survival in node-positive patients with endometrial cancer. Gynecol Oncol 2005;96:651-2.

19. Ballouk F, Ross JS, Wolf BC. Ovarian endometriotic cysts. An analysis of cytologic atypia and DNA ploidy patterns. Am J Clin Pathol 1994;102:415-9.

20. Jimbo H, Hitomi Y, Yoshikawa H, Yano T, Momoeda $\mathrm{M}$, Sakamoto $A$, et al. Evidence for monoclonal expansion of epithelial cells in ovarian endometrial cysts. Am J Pathol 1997;150:1173-8.

21. Thomas EJ, Campbell IG. Molecular genetic defects in endometriosis. Gynecol Obstet Invest 2000;50(Suppl 1):44-50.

22. Moinfar F, Gogg-Kamerer M, Sommersacher A, Regitnig P, Man YG, Zatloukal K, et al. Endometrial stromal sarcomas frequently express epidermal growth factor receptor (EGFR, HER-1): potential basis for a new therapeutic approach. Am J Surg Pathol 2005;29:4859.

23. Lacroix-Triki M, Beyris L, Martel P, Marques B. Lowgrade endometrial stromal sarcoma arising from sciatic nerve endometriosis. Obstet Gynecol 2004;104(5 Pt 2):1147-9.

24. Mitchard JR, Lott M, Afifi RA, Hirschowitz L. Low-grade endometrial stromal sarcoma with glandular differentiation arising in ovarian endometriosis. J Obstet Gynaecol 2004;24:596-7.

25. Bodner K, Zauner M, Bodner-Adler B, Spangler B, Grunberger W, Wierrani F. Parenchymatous pulmonary endometriosis-metastases of a low-grade endometrial stromal sarcoma? Med Hypotheses 2003;61:651-3. 